\title{
Variable fitness effects of Wolbachia infection in Drosophila melanogaster
}

\author{
AJ Fry, MR Palmer and DM Rand \\ Department of Ecology and Evolutionary Biology, Box G-W, Brown University, Providence, RI 02912, USA
}

\begin{abstract}
Maternally inherited Wolbachia bacteria are extremely widespread among insects and their presence is usually associated with parasitic modifications of host fitness. Wolbachia pipientis infects Drosophila melanogaster populations from all continents, but their persistence in this species occurs despite any strong parasitic effects. Here, we have investigated the symbiosis between Wolbachia and $D$. melanogaster and found that Wolbachia infection can have significant survival and fecundity effects. Relative to uninfected flies, infected females from three fly strains showed enhanced survival or fecundity associated with Wolbachia infection, one strain showed both and one strain responded positively to Wolbachia removal. We found no difference in egg hatch rates (cytoplasmic incompatibility) for crosses between infected males and uninfected females, although there were fecundity differences. Females from this cross consistently produced fewer eggs than infected females and
\end{abstract}

these fecundity differences could promote the spread of infection just like cytoplasmic incompatibility. More surprising, we found that infected females often had the greatest fecundity when mated to uninfected males. This could also promote the spread of Wolbachia infection, though here the fitness benefits would also help to spread infection when Wolbachia are rare. We suggest that variable fitness effects, in both sexes, and which interact strongly with the genetic background of the host, could increase cytoplasmic drive rates in some genotypes and help explain the widespread persistence of Wolbachia bacteria in $D$. melanogaster populations. These interactions may further explain why many $D$. melanogaster populations are polymorphic for Wolbachia infection. We discuss our results in the context of host-symbiont co-evolution.

Heredity (2004) 93, 379-389. doi:10.1038/sj.hdy.6800514

Published online 11 August 2004

Keywords: endosymbiosis; fecundity; mutualism; parasitism; survival; symbiosis

\section{Introduction}

Vertical transmission and an endosymbiotic lifestyle will favor the evolution of mutualistic interactions between bacteria and their hosts (Anderson and May, 1982; Bull et al, 1991; Lipsitch et al, 1995). As mutualistic endosymbionts are often maternally inherited, natural selection should favor endosymbionts that improve the fitness (survival and fecundity) of female hosts because they transmit the endosymbionts (Turelli, 1994). Wolbachia is a genus of maternally inherited $\alpha$-Proteobacteria that form a monophyletic group with pathogenic Ehrlichia and Rickettsia bacteria (Anne-Veronique et al, 2003). Wolbachia are extremely widespread among arthropods and may infect as many as $76 \%$ of all insect species (Werren et al, 1995a; Jeyaprakash and Hoy, 2000). As Wolbachia are maternally inherited, they should provide fitness benefits to their hosts and in some cases they do. For example, in nematode worms (Bandi et al, 1999) and a parasitic wasp (Dedeine et al, 2001), Wolbachia infection is necessary for normal host reproduction. However, these mutualistic examples are considered atypical because Wolbachia usually manipulate their host's reproduction in ways that enhance bacterial transmission. Wolbachia's repro-

Correspondence: AJ Fry, Current address: Marine Biological Laboratory, 7 MBL Street, Woods Hole, MA 02543, USA. E-mail: afry@mbl.edu Received 23 May 2003; accepted 6 May 2004; published online 11 August 2004 ductive manipulations often have a fitness cost to male hosts, which can be genetically transformed into females, or killed, when Wolbachia are present (reviewed in Stouthamer et al, 1999). One explanation for the widespread occurrence of maternally inherited and parasitic Wolbachia is that Wolbachia must undergo some horizontal transmission among hosts. Such horizontal movement between suitable host species has been well documented in the laboratory and inferred from phylogenetic reconstructions of different Wolbachia genes (Werren et al, 1995b).

In the vinegar fly genus Drosophila, Wolbachia causes an egg mortality phenotype known as cytoplasmic incompatibility (CI). CI has been studied in several taxa including D. simulans (eg, Hoffmann et al, 1986, 1990; Hoffmann and Turelli, 1988) and D. melanogaster (Hoffmann et al, 1994, 1998; Solignac et al, 1994). CI is manifest as severe egg mortality (up to 95\%) when an infected male mates with an uninfected female (reviewed in Werren, 1997). This phenotype provides indirect fitness benefits to infected females and allows Wolbachia bacteria to colonize a host population (Caspari and Watson, 1959; Fine, 1978; Turelli, 1994). In D. melanogaster, CI strength is surprisingly variable (Solignac et al, 1994) and often weak (Hoffmann et al, 1994, 1998) compared to the strong incompatibility induced by closely related Wolbachia strains in D. simulans (Hoffmann et al, 1990). Despite weak and variable CI strength, Wolbachia are present in D. melanogaster populations from all continents. This widespread distribution could reflect an ancient 
historical association between $D$. melanogaster and Wolbachia, which predates the emergence of $D$. melanogaster from Africa and into its current geographic distribution. However, the observations of weak and variable CI in $D$. melanogaster and the widespread occurrence of Wolbachia among D. melanogaster populations has led to the suggestion that Wolbachia must confer some fitness advantage to $D$. melanogaster females (Hoffmann et al, 1994, 1998; Solignac et al, 1994). To investigate this, Hoffmann et al (1994, 1998) compared infected and uninfected flies for laboratory fecundity, sperm competition, fluctuating asymmetry, and thorax length measured from field-collected flies. They found no detectable fitness effects attributable to Wolbachia infection. However, several recent studies have suggested some potential symbiont effects. Reynolds and Hoffmann (2002) showed that CI induction in D. melanogaster could be very strong in young males, which could help explain the persistence of Wolbachia if wild males mate when they are 1-2 days old. In addition, Olsen et al (2001) described positive fecundity effects associated with Wolbachia infection in Australian D. melanogaster, but these effects depended on the nuclear background of the host.

We investigated Wolbachia's effects on D. melanogaster fitness for three reasons. First, Min and Benzer (1997) described an unusual Wolbachia strain, called wDmpopcorn, which severely reduced $D$. melanogaster survival. We tested $D$. melanogaster for survival effects associated with its naturally occurring Wolbachia strain, called $w \mathrm{Dm}$. Second, some Wolbachia effects, like CI and bacterial density, are known to decline with male host age (eg, Hoffmann et al, 1986), and there may be age-specific survival or fecundity effects. Third, Wolbachia affects host reproduction and there is often a negative correlation between survival and certain aspects of reproduction (eg, Fowler and Partridge, 1989; Chapman et al, 1998). We measured survival, egg to adult viability, fecundity, and the strength of cytoplasmic incompatibility for all crosses between infected and uninfected $D$. melanogaster flies. Our experiments were conducted in the laboratory with large sample sizes to reduce uncontrolled environmental variation. We also included an uninfected treatment control strain to test for effects of antibiotic treatment to remove Wolbachia bacteria. We found evidence for both positive and negative fitness effects associated with Wolbachia bacteria. Our results suggest the presence of segregating fitness variation associated with the Wolbachia pipientis-Drosophila melanogaster symbiosis. We suggest that this segregating variation could help explain the persistence of Wolbachia bacteria in D. melanogaster and also suggests that normally parasitic Wolbachia might have neutral or even beneficial effects in some insect hosts.

\section{Materials and methods}

\section{Drosophila strains, antibiotic treatment, and DNA sequencing}

We used five $D$. melanogaster laboratory strains that carry the endosymbiotic bacterium $W$. pipientis. They are Z53 and Z2, both from Zimbabwe Africa, and were collected at the Sengwa Wildlife Reserve in 1990 (see Wu et al, 1995). The other strains, Ftf1 and Ftf100, were both collected in 1992 from Four-Town Farm in RI, USA. A fifth laboratory strain, WJ9, was provided by Marc Tatar (Brown University). These laboratory strains were started as isofemale lines, have been in laboratory culture for several hundred generations, and are highly inbred. All Wolbachia strains used in this study share identical 16S rDNA sequences, and share identical sequences at wsp, one of the most highly expressed and variable Wolbachia genes (Braig et al, 1998). To create genetically similar infected and uninfected fly lines from a single infected stock, we used an antibiotic treatment protocol that has been widely employed (eg, Hoffmann et al, 1994; Poinsot and Mercot, 1997; Bordenstein and Werren, 1998; Dobson et al, 2002). We created uninfected or treated (T) fly lines by adding $0.25 \mathrm{mg} / \mathrm{ml}$ of the antibiotic tetracycline in water to Carolina dry food in a 1:1 mix. We did this for two discrete generations to ensure that Wolbachia were completely removed. Our Wolbachia-infected (W) lines received identical food and environment as the $\mathrm{T}$ lines, except that tetracycline was not added. After treatment, we confirmed the infection status of all lines using Wolbachia-specific 16S rDNA PCR primers (O'Neill et al, 1992). Individual PCR was performed on at least 25 flies from each $\mathrm{T}$ and $\mathrm{W}$ line to insure against contamination of treated stocks and to insure that infected stocks remained infected. PCR checks were also carried out at each generation after treatment to insure the continued status of $\mathrm{T}$ and $\mathrm{W}$ lines.

To ensure that differences between $\mathrm{T}$ and $\mathrm{W}$ lines were not due to tetracycline treatment, three procedures were used. First, we treated an uninfected fly strain, RIredE, to determine the affect of tetracycline treatment on its survival. Second, because Clancy and Hoffmann (1998) have shown that tetracycline treatment can depress Drosophila fitness, we held our flies for two generations on tetracycline-free food after treatment, but before the experiments. This was done to minimize the maternal effects of treatment. Third, fecundity was assayed from two fly strains at two different times (two and ten generations after treatment). We did this because previous studies have shown that treatment to remove CI expressing Wolbachia can have temporary fitness effects on some hosts, like Nasonia vitripennis (Bordenstein and Werren, 2000) and D. simulans (Poinsot and Mercot, 1997). Unlike these studies, however, D. melanogaster has only one Wolbachia strain that does not induce strong CI. All fly strains used in this study were maintained in population cages that hold six food bottles and approximately 1000 adult flies. Flies were maintained in this way for 10 generations prior to tetracycline treatment, during treatment, and for all generations following treatment. To control larval density at each generation, we allowed adult flies to deposit approximately 120-150 eggs in each food bottle to initiate the next generation.

\section{Survival}

At the start of the third generation post-treatment, we measured age-specific survival of $\mathrm{T}$ and $\mathrm{W}$ lines by placing 100 virgin males and 100 virgin females into three replicate demography cages. The demography cages were made from quart-serving plastic containers, with a screened lid, and a side coupling of the same dimension as a standard food vial. The cages were placed in a walk-in incubator and kept on a 12L:12D 
photoperiod at $25^{\circ} \mathrm{C}$ and $40 \%$ relative humidity. The food vials were replaced every other day and dead flies were removed with an aspirator, sexed and counted. The cages were monitored in this way until nearly all the flies had died. Survival was analyzed with a semiparametric proportional hazards statistical model (Cox, 1972). In this model, our dependent variable was the time of death measured to the nearest $48 \mathrm{~h}$, with fly strain (Z53, Z2, Ftf1, Ftf100, and WJ9), infection status (T or W), sex (male or female), and their interactions as predictors. Survival curves from $\mathrm{T}$ and $\mathrm{W}$ lines were compared using logrank tests.

We estimated age-specific mortality models for $\mathrm{T}$ and $W$ lines using maximum likelihood because linear regression of log-mortality rates on age can produce biased estimates of mortality model parameters (Mueller et al, 1995; Promislow et al, 1997; Pletcher, 1999). Four mortality models were considered (Vaupel and Yashin, 1985). The Gompertz (G) model describes an exponential increase in mortality rate with age and is given by $\mu_{(x)}=\alpha \mathrm{e}^{\beta(x)}$, where $\mu_{(x)}$ is the mortality rate at age $x, \alpha$ is the initial mortality rate or intercept, and $\beta$ is the slopeor age-dependent increase in mortality. The GompertzMakeham (GM) model is the Gompertz plus a parameter, $C$, that describes age-independent mortality. The logistic (L) model is the Gompertz plus a frailty parameter, s, to describe late-life mortality rate deceleration. The Makeham and logistic models can be combined with the Gompertz to produce a four-parameter logistic-Makeham (LM) model. Since these models are nested, likelihood ratio tests were used to compare model parameters from $\mathrm{T}$ and $\mathrm{W}$ lines. We partitioned the total difference in mortality between $\mathrm{T}$ and $\mathrm{W}$ lines into the additive contributions of each parameter to understand which parameters contributed most to the overall mortality difference (Pletcher et al, 2000).

\section{Fecundity and cytoplasmic incompatibility}

Fecundity was measured at the start of the third generation post-treatment. We measured fecundity from all experimental strains except WJ9 because the infected stock was accidentally treated following the survival assays. For each fly strain, four crosses were made and are numbered throughout this report as follows: (1) W male $\times \mathrm{T}$ female, (2) T male $\times \mathrm{W}$ female, (3) $\mathrm{T}$ male $\times \mathrm{T}$ female, (4) W male $\times W$ female. Each cross was replicated 50 times. For each cross, a newly eclosed single female and two same-aged males were placed into food vials to mate and lay eggs. If one of the males died, it was replaced with an appropriately aged male to insure continued access to sperm. The flies were transferred to fresh food vials at the same time each day and the number of eggs deposited in the food vials during the previous $24 \mathrm{~h}$ was recorded. For strains Ftf100 and Z2, eggs were collected during a $24 \mathrm{~h}$ period, $16 \mathrm{~h}$ after eclosion, and $8 \mathrm{~h}$ after mating. A second fecundity measure was taken on these strains at 5 days posteclosion. For two fly strains, Z53 and Ftf1, we measured the lifetime fecundity by counting eggs every day (Z53) or every other day (Ftf1) until all the females had died.

CI was measured at the start of the third generation post-treatment. CI exists when the proportion of eggs hatching from the incompatible cross (cross 1) is significantly less than for compatible crosses (crosses
2-4). CI was measured by holding the egg-counting vials from the fecundity assays for an additional 35-40 h when the number of unhatched eggs in each vial was recorded and compared with the previous egg counts. For strains Ftf1 and Z53, CI was measured 7 days after females had mated. For all the four strains, CI was measured with young males during a $24 \mathrm{~h}$ period, $16 \mathrm{~h}$ after males and females had eclosed, and $8 \mathrm{~h}$ after mating. This was done to allow comparison with two fly strains reported in a previous study of CI in D. melanogaster (Weeks et al, 2002).

\section{Viability}

Larval to adult viability was measured at the start of the third generation post-treatment and was scored for each fly strain from eggs deposited by females during a $24 \mathrm{~h}$ period, $16 \mathrm{~h}$ after eclosion, and $8 \mathrm{~h}$ after mating. The protocol was to hold food vials containing eggs for 14 days after egg laying and count the number of emerging adults. The number of emerging adults was then compared with the number of hatched eggs recorded previously for that vial. As for fecundity and $\mathrm{CI}$, we started with 50 female replicates for each of the four crosses.

\section{Results}

\section{Survival}

The proportional hazards analysis revealed strong effects of treatment to remove Wolbachia, fly strain, and sex on survival as well as significant interactions between and among them (Table 1). As there were no significant differences between the replicate cages for any of the factors investigated, those data are pooled and replicate cage is not considered a factor in the analysis. Wolbachia infection produced a strong and significant survival response in three of five D. melanogaster strains (see risk ratios in Table 2). Two strains, Z53 and Ftf1, lived longer when infected with Wolbachia (Figure 1), although these differences were age dependent. A survival response was also seen in females and Ftf1 males (not shown). Infected Z53 males lived longer than uninfected Z53 males (Figure 1), but the difference was not significant $(P=0.06)$. One fly strain, WJ9, showed decreased survival (Figure 2) associated with Wolbachia infection as both males and females survived approximately $12 \%$ longer than infected flies (Table 2). The two remaining experimental fly strains, Z2 and Ftf100, showed no survival effect associated with Wolbachia or tetracycline

Table 1 Proportional hazards analysis of variance for survival

\begin{tabular}{lcrc}
\hline Source & d.f. & $L-R \chi^{2}$ & $\mathrm{P}$ \\
\hline Fly strain (FS) & 4 & 2482.1 & $<10^{-4}$ \\
Sex & 1 & 511.8 & $<10^{-4}$ \\
Treatment & 1 & 119.3 & $<10^{-4}$ \\
FS $\times$ Sex & 4 & 389.5 & $<10^{-4}$ \\
Treatment $\times$ FS & 4 & 132.5 & $<10^{-4}$ \\
Treatment $\times$ Sex & 1 & 1.3 & $=0.24$ \\
Treatment $\times$ FS $\times$ Sex & 4 & 25.2 & $<10^{-4}$ \\
\hline
\end{tabular}

Main effects are fly strain (Z53, Z2, Ftf1, Ftf100, WJ9), sex (male or female), and treatment ( $\mathrm{T}$ or $\mathrm{W})$. Likelihood ratio $\chi^{2}$ value and associated probability are given. 
Table 2 Summary of survival and mortality analyses

\begin{tabular}{|c|c|c|c|c|c|c|c|c|c|c|}
\hline \multirow[t]{2}{*}{ Fly strain } & \multicolumn{5}{|c|}{ Female } & \multicolumn{5}{|c|}{ Male } \\
\hline & Z53 & Ftf1 & WJ9 & Ftf100 & $\mathrm{Z2}$ & Z53 & Ftf1 & WJ9 & Ftf100 & $\mathrm{Z2}$ \\
\hline Survival effect & + & + & - & + & + & + & + & - & + & + \\
\hline Risk ratio $\mathrm{W} / \mathrm{T}$ & 1.25 & 1.15 & 0.88 & 1.03 & 1.01 & 1.08 & 1.34 & 0.89 & 1.04 & 1.06 \\
\hline Log-rank $\chi^{2}$ & 52.28 & 23.36 & 11.31 & 1.21 & 0.05 & 3.61 & 135.10 & 10.30 & 1.41 & 2.58 \\
\hline$p$ & $<10^{-3}$ & $<10^{-3}$ & $<10^{-2}$ & 0.27 & 0.81 & 0.06 & $<10^{-3}$ & $<10^{-2}$ & 0.23 & 0.11 \\
\hline \multicolumn{11}{|l|}{ Mortality model } \\
\hline Wolbachia & G,GM & G,GM & G & L,LM & G & GM,LM & G & $\mathrm{L}, \mathrm{LM}$ & L,LM & G \\
\hline Treated & G & G,GM & LM & L,LM & G & GM,LM & G,GM & L,LM & L,LM & G \\
\hline$\alpha$ & 0.93 & 13.28 & 63.79 & & & & 76.06 & 98.80 & & \\
\hline$\beta$ & 98.58 & 85.94 & 34.54 & & & & 23.70 & & & \\
\hline C & 0.49 & 0.78 & 1.17 & & & & 0.24 & & & \\
\hline$s$ & & & 0.50 & & & & & 1.20 & & \\
\hline
\end{tabular}

All entries compare Wolbachia-infected (W) and tetracycline-treated (T) D. melanogaster strains. For each fly strain and sex, Wolbachia's positive $(+)$ or negative (-) survival effect is indicated. The risk ratio compares the risk of death or hazard. Survival curves were compared with logrank tests. $\chi^{2}$ and associated $P$-values reported. Mortality models (see text) with the highest likelihood scores are indicated. In many cases, two mortality models fit the data equally well. Entries for $\alpha, \beta, C$, and $s$ give the percent contribution of that model parameter to the total difference in mortality between $\mathrm{W}$ and $\mathrm{T}$ lines for a given fly strain and sex.

treatment $(P>0.05)$ (Table 2). Neither males nor females from the uninfected control strain, RiRedE, responded to tetracycline treatment or Wolbachia removal $(P>0.05)$, suggesting that tetracycline treatment itself was an unlikely cause for the survival effects we observed.

Age-specific mortality rates of $\mathrm{T}$ and $\mathrm{W}$ lines are shown in Figures 1 and 2. Mortality curves are plotted as $\left[-\ln \left(N_{t+1} / N_{t}\right)\right]$ versus age, where $N_{t}$ and $N_{t+1}$ are the numbers of individuals alive during consecutive time intervals. For $\mathrm{T}$ and $\mathrm{W}$ lines the best-fitting mortality model was estimated, and for each mortality curve the intercept $(\alpha)$, slope $(\beta)$, Makeham constant $(C)$, and frailty parameter $(s)$ were estimated using maximum likelihood (Pletcher, 1999). The $\mathrm{T}$ and $\mathrm{W}$ mortality curves were different for females from three strains, Z53, Ftf1, and WJ9. Only WJ9 showed a significant difference in best-fit mortality model (see Table 2). In WJ9, the mortality curve of $\mathrm{T}$ females is best described by a Gompertz model, whereas a logistic-Makeham model is best for W females. The bottom of Table 2 gives the percent contribution of each mortality model parameter to the total difference in mortality observed between $\mathrm{T}$ and $\mathrm{W}$ lines. The females from WJ9 differ primarily in the rate of aging $(\beta), 35 \%$, and in the initial mortality rate $(\alpha), 64 \%$, but the parameters $C$ and $s$, which account for the different best-fit mortality models, contributed very little $(<2 \%)$. In Z53 and Ftf1, the mortality differences between T and $\mathrm{W}$ lines were due to differences in the intercept $(\alpha)$ and slope $(\beta)$ of the mortality models.

\section{Fecundity and cytoplasmic incompatibility}

Three of four Drosophila strains showed a significant decrease in fecundity after Wolbachia were removed with antibiotics. Infected (W) females from Ftf1 and Ftf100 produced significantly more eggs than uninfected $(\mathrm{T})$ females (ie, crosses 2 and $4>$ crosses 1 and 3 ) during time 1 (Table 3). The African fly strains Z53 and Z2 did not show a fecundity effect during this time or during time 2 . However, the analysis of lifetime fecundity revealed that, in Z53, cross 2 produced significantly more eggs than cross 1, the 'CI cross' (although no CI was detected, see below). This pattern is evident in Figure 3. By dividing the first 20 days of egg laying into 5-day intervals, we find that between days 6 and 15 (Figure 3, panels B and C) Z53 shows the same pattern of increased fecundity, as does Ftf1. In Z53, cross 2 was significantly more fecund than cross 1 during all time periods, even though traditional comparisons of uninfected and infected mating (cross 3 versus 4) would not find differences during the first 5 days or the last 5 days (Figure 3, panels A, D).

During the third generation following tetracycline treatment, we tested our fly strains for CI at two egglaying times: right after eclosion and 1 week later. The first occurred during a $24 \mathrm{~h}$ egg-laying period beginning $16 \mathrm{~h}$ after eclosion (strains Ftf1, Ftf100, Z53, Z2) and the second during another $24 \mathrm{~h}$ collection period occurring 7 days after eclosion (Ftf1, Z53). Contrary to an earlier report of strong CI induction when infected males are very young (Weeks et al, 2002), we found no evidence for early CI as there were no significant differences in egg hatches among the four crosses (Table 4, time 1). No significant hatch differences were detected at 7 days post-eclosion either. Unlike the Weeks et al (2002) study, we found that egg hatch proportions were higher, not lower, early in life (Table 4, compare Ftf1 and Z53 times 1 and 2), indicating that $\mathrm{CI}$ is not present in our fly strains. Furthermore, our data indicate that strong and early CI cannot be a general phenomenon among Wolbachia-infected D. melanogaster fly strains (Reynolds and Hoffmann, 2002).

\section{Viability}

Viability was also measured on flies three generations removed from tetracycline treatment. We measured the egg to adult viability by counting the number of emerging adults 14 days after eggs were deposited into food vials (Table 5). There were no significant differences in the proportions of hatched eggs developing into adults for any of the fly strains except Ftf1. In Ftf1, the viability of cross $3(\mathrm{~T} \times \mathrm{T})$ was significantly less than cross 2 (T male $\times \mathrm{W}$ female). However, due to the 'ideal' laboratory conditions in which our flies were reared, the viability of strains Ftf1, Ftf100, and Z53 was high, about 

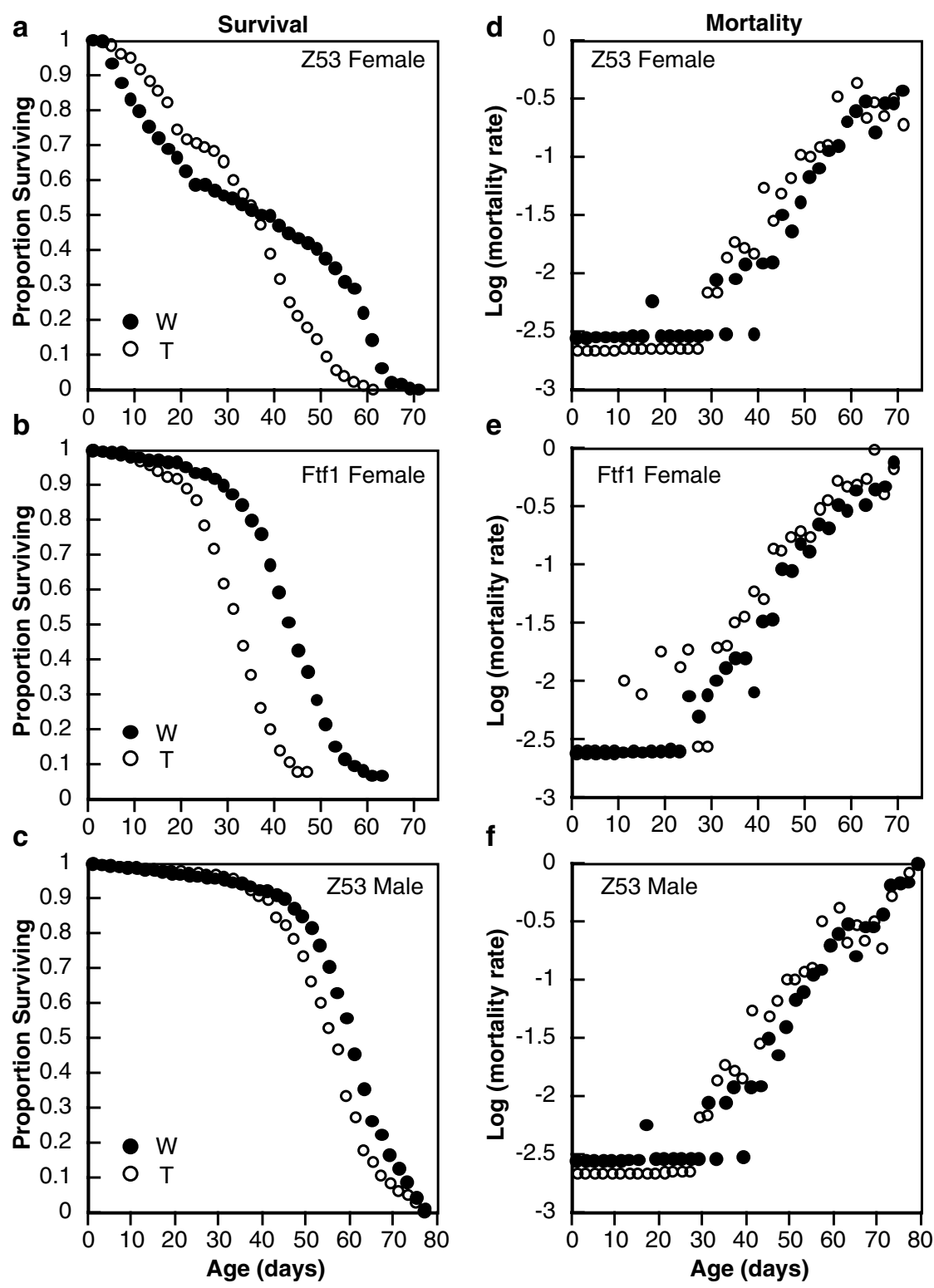

Figure 1 Survival (a-c) and mortality (d-f) of Wolbachia-infected (W) and tetracycline-treated (T) D. melanogaster. Survival curves compared with log-rank tests (see Table 2). Mortality rates are $\left(-\ln \left(N_{t+1} / N_{t}\right)\right)$, where $N_{t}$ and $N_{t+1}$ are the number of individuals alive during consecutive time intervals. Mortality parameters were compared with likelihood ratio tests (Table 2).

$85 \%$ for the four crosses, but was about $10 \%$ less $(75 \%)$ for strain Z2 (Table 5). Previous crossing experiments have suggested that strain $\mathrm{Z} 2$ carries deleterious mutations for fitness characters (Fry and Rand, 2002).

\section{Discussion}

There was no evidence for cytoplasmic incompatibility in our D. melanogaster fly strains. We tested $\mathrm{CI}$ at two times: $\mathrm{CI}$ was measured using adult flies that had eclosed $16 \mathrm{~h}$ earlier, and CI was measured again on the same fly strains when the imagoes were 7 days old. Our results contrast with an earlier report of strong CI when infected $D$. melanogaster males are young (Reynolds and Hoffmann, 2002). We found no evidence for early CI at any time, as there were no significant differences in egg hatch among the four crosses (Table 4). In previous studies of CI in D. melanogaster (eg, Weeks et al, 2002; Reynolds and Hoffmann, 2002), the age of the females used is not clear, nor is there mention of whether unhatched eggs had received a complement of paternal chromosomes. In this report, CI was measured with males and females of nearly identical age, and we did not check unhatched eggs for paternal chromosomes because egg hatch proportions were generally high and did not differ among the crosses (Table 4). Our data from four Drosophila strains, representing both North American and African populations, do not support the generality of strong or early cytoplasmic incompatibility in D. melanogaster. Clearly, additional D. melanogaster strains must be examined to determine if early and strong CI can explain the persistence of Wolbachia infection among worldwide populations of D. melanogaster. 

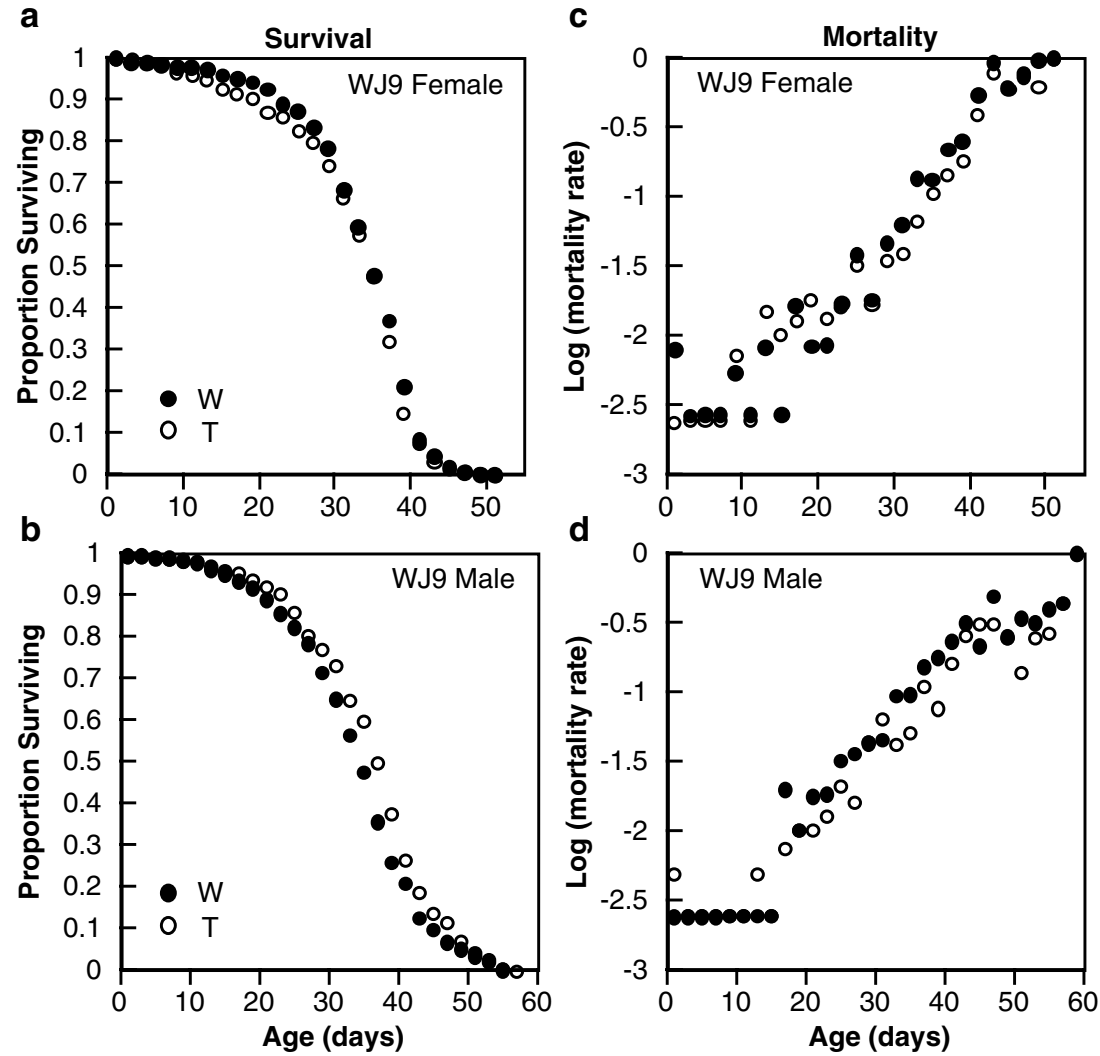

Figure 2 Survival (a) and mortality (b) from D. melanogaster strain WJ9. Survival and mortality rates were calculated as in Figure 1. T and W survival was significantly different (Table 2 ).

Table 3 Fecundity - the mean number of eggs from each of four crosses $(\mathrm{W} \times \mathrm{T}=$ Wolbachia male crossed to treated female)

\begin{tabular}{|c|c|c|c|c|}
\hline Fly strain & Cross $1(W \times T)$ & Cross2 $(T \times W)$ & Cross $3(T \times T)$ & Cross $4(W \times W)$ \\
\hline \multicolumn{5}{|l|}{ Time 1} \\
\hline Ftf1 & $16.2^{\mathrm{a}}(47,1.2)$ & $25.7^{\mathrm{b}}(45,1.3)$ & $18.2^{\mathrm{a}}(46,1.2)$ & $25.6^{\mathrm{b}}(50,1.5)$ \\
\hline Ftf100 & $12.2^{\mathrm{a}}(39,1.6)$ & $23.1^{\mathrm{b}}(48,1.4)$ & $16.5^{\mathrm{a}}(41,2.1)$ & $23.1^{\mathrm{b}}(48,1.2)$ \\
\hline Z53 & $26.1^{\mathrm{a}}(47,1.3)$ & $26.2^{\mathrm{a}}(47,1.9)$ & $29.5^{\mathrm{a}}(46,2.3)$ & $30.3^{\mathrm{a}}(39,2.8)$ \\
\hline $\mathrm{Z} 2$ & $17.9^{a}(41,1.3)$ & $16.4^{\mathrm{a}}(48,1.3)$ & $14.9^{\mathrm{a}}(39,1.2)$ & $17.4^{\mathrm{a}}(49,1.2)$ \\
\hline \multicolumn{5}{|l|}{ Time 2} \\
\hline Ftf100 & $23.7^{\mathrm{a}}(38,1.4)$ & $26.4^{\mathrm{a}}(43,1.2)$ & $27.9^{\mathrm{a}}(37,1.4)$ & $26.1^{\mathrm{a}}(43,1.1)$ \\
\hline $\mathrm{Z} 2$ & $42.3^{a}(41,1.8)$ & $41.1^{\mathrm{a}}(48,1.6)$ & $42.1^{\mathrm{a}}(39,2.0)$ & $39.3^{\mathrm{a}}(49,1.7)$ \\
\hline \multicolumn{5}{|l|}{ Lifetime } \\
\hline Ftf1 & $374.4^{\mathrm{a}}(40,35.2)$ & $555.8^{\mathrm{b}}(50,31.9)$ & $404.5^{\mathrm{a}}(44,30.5)$ & $566.4^{\mathrm{b}}(50,24.1)$ \\
\hline Z53 & $692.9^{\mathrm{a}}(50,48.5)$ & $903.9^{\mathrm{b}}(50,43.8)$ & $712.7^{\mathrm{ab}}(48,47.7)$ & $769.9^{\mathrm{ab}}(50,67.1)$ \\
\hline
\end{tabular}

Entries are the mean number of eggs laid (sample size, SE). Time $1=24 \mathrm{~h}$ collection period $16-40 \mathrm{~h}$ after eclosion. Time $2=24 \mathrm{~h}$ collection period at 5 days post-eclosion. Lifetime fecundity (see text). Crosses compared with Tukey-Kramer HSD tests. Significant differences among the crosses within a strain are indicated by different letters.

In the absence of reproductive modifications such as cytoplasmic incompatibility, Wolbachia should be lost from $D$. melanogaster host populations. This is true unless Wolbachia infection confers some fitness advantage to infected females or manipulates host reproduction in ways that are not yet appreciated. Our results indicate that Wolbachia bacteria can have variable affects on $D$. melanogaster fitness. After antibiotic treatment to remove Wolbachia, uninfected flies from strain WJ9 survived significantly longer than infected flies (Figure 2). Furthermore, WJ9 females were the only strain to show a difference in best-fit mortality model between $\mathrm{T}$ and $\mathrm{W}$ flies. One interpretation from Table 2 is that Wolbachia infection has removed a source of age-independent mortality (C) and reduced the frailty (s) of infected females relative to uninfected females, even though the latter survived longer. However, these two parameters together contributed less than $4 \%$ to the total difference in mortality between WJ9 T and W flies. It is worth noting that the statistical power to detect differences in the parameters $C$ and $s$ is strongly dependent on sample size. There is low power at the beginning of the survival experiments because few individuals die at the earliest ages $(C)$, and there is also low power at the end of the 

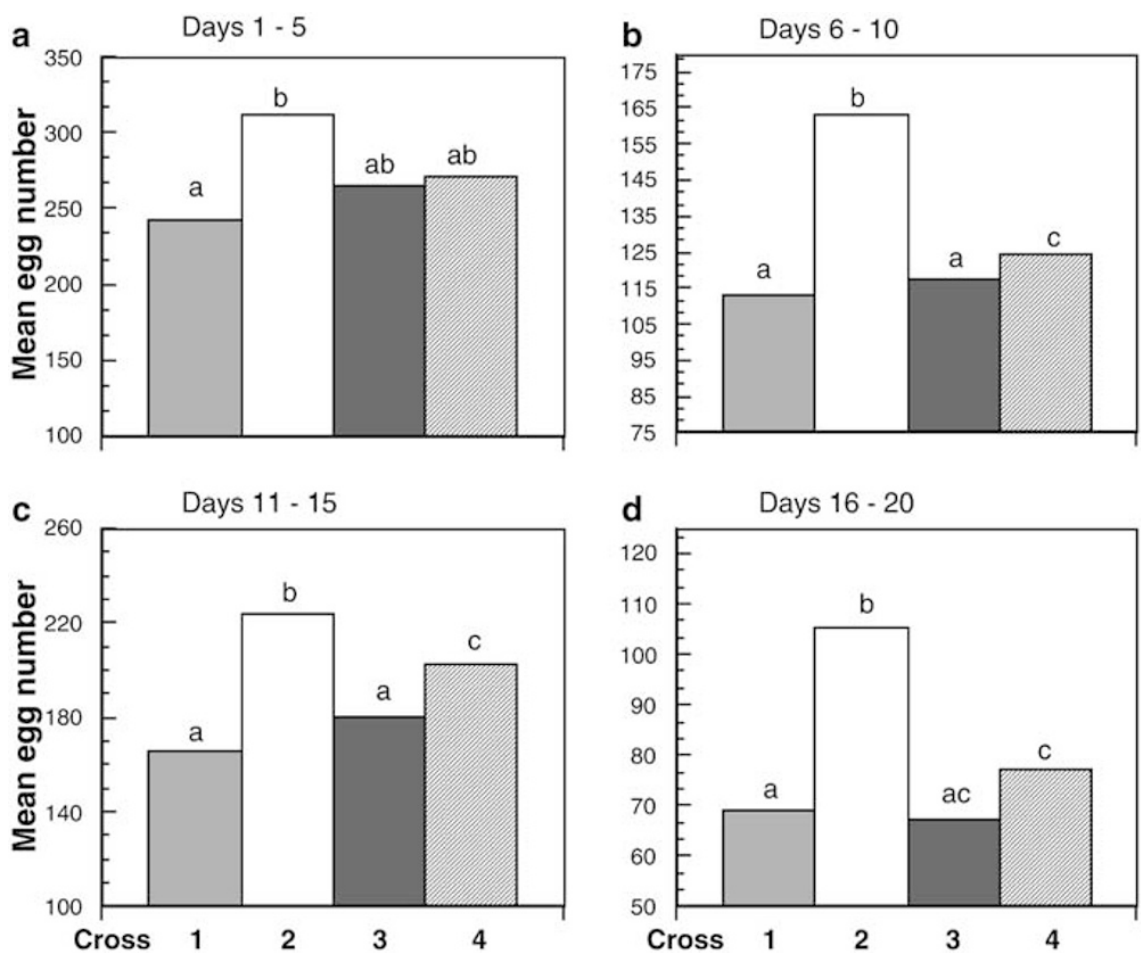

Figure 3 Age-specific fecundity from Z53 females divided into four consecutive 5-day intervals. Data are from time 1. The mean number of eggs laid by $48-50$ females per cross. See Materials and methods for cross definitions. Means compared with Tukey-Kramer HSD. Different letters above histogram bars indicate significant differences.

Table 4 Cytoplasmic incompatibility

\begin{tabular}{|c|c|c|c|c|}
\hline Fly strain & Cross $1(W \times T)$ & Cross2 $(T \times W)$ & Cross $3(T \times T)$ & Cross $4(W \times W)$ \\
\hline \multicolumn{5}{|l|}{ Time 1} \\
\hline Ftf1 & $97.5(47,0.04)$ & $97.9(45,0.03)$ & $96.7(46,0.10)$ & $97.3(50,0.04)$ \\
\hline Ftf100 & $95.4(39,0.07)$ & $94.4(48,0.05)$ & $96.7(41,0.06)$ & $94.5(48,0.05)$ \\
\hline Z53 & $94.1(47,0.07)$ & $90.5(47,0.06)$ & $92.1(46,0.13)$ & $91.6(38,0.14)$ \\
\hline $\mathrm{Z} 2$ & $81.3(41,0.23)$ & $78.6(48,0.21)$ & $78.5(39,0.22)$ & $80.9(49,0.19)$ \\
\hline \multicolumn{5}{|l|}{ Time 2} \\
\hline Ftf1 & $74.3(30,0.47)$ & $86.2(47,0.34)$ & $72.6(40,0.46)$ & $77.8(50,0.46)$ \\
\hline Z53 & $90.1(50,0.11)$ & $83.3(50,0.15)$ & $83.0(50,0.14)$ & $90.2(50,0.16)$ \\
\hline
\end{tabular}

The proportion of eggs hatching from each of four crosses $(\mathrm{W} \times \mathrm{T}=$ Wolbachia male crossed to treated female). Entries are the mean proportion of eggs hatching (sample size, SE). Time $1=24 \mathrm{~h}$ sampling period $16-40 \mathrm{~h}$ after eclosion. Time $2=24 \mathrm{~h}$ collection period at 7 days post-eclosion. There were no significant differences among the crosses within any strain.

Table 5 Viability - the proportion of adults emerging as a proportion of eggs that hatched 14 days earlier $(\mathrm{W} \times \mathrm{T}=$ Wolbachia male mated to treated female)

\begin{tabular}{lccrr}
\hline Fly strain & Cross 1 $(W \times T)$ & Cross 2 $(T \times W)$ & Cross 3 $(T \times T)$ & Cross $4(W \times W)$ \\
\hline Ftf1 & $86.7(47,0.17)$ & $88.1(45,0.09)$ & $79.1(46,0.18)$ & $83.9(50,0.16)$ \\
Ftf100 & $87.7(38,0.17)$ & $90.2(48,0.13)$ & $85.9(41,0.15)$ & $88.5(48,0.16)$ \\
Z53 & $86.7(47,0.11)$ & $87.7(47,0.10)$ & $87.9(46,0.09)$ & $84.1(38,0.13)$ \\
Z2 & $81.1(40,0.22)$ & $76.5(47,0.25)$ & $69.8(38,0.26)$ & $75.4(48,0.24)$ \\
\hline
\end{tabular}

Entries are means (sample size, SE). Eggs collected during a $24 \mathrm{~h}$ period, 16-40 h after eclosion. Only crosses 2 and 3 from Ftf1 are significantly different by Tukey-Kramer HSD.

survival experiments because there are few individuals left to die at the latest ages (s). Therefore, our power to detect differences in $C$ and $s$ may be less than for the slope $(\beta)$ and intercept $(\alpha)$. That the differences in WJ9 females are significant by likelihood ratio tests suggests that Wolbachia's negative effects on fitness could be produced differently than Wolbachia's positive effects on fitness (Table 2). Negative fitness effects, like those we found in strain WJ9, have been reported previously in Drosophila. For example, in the closely related $D$. simulans, Hoffmann et al (1990) found that Wolbachia produced a $10-20 \%$ reduction in female fecundity. In 


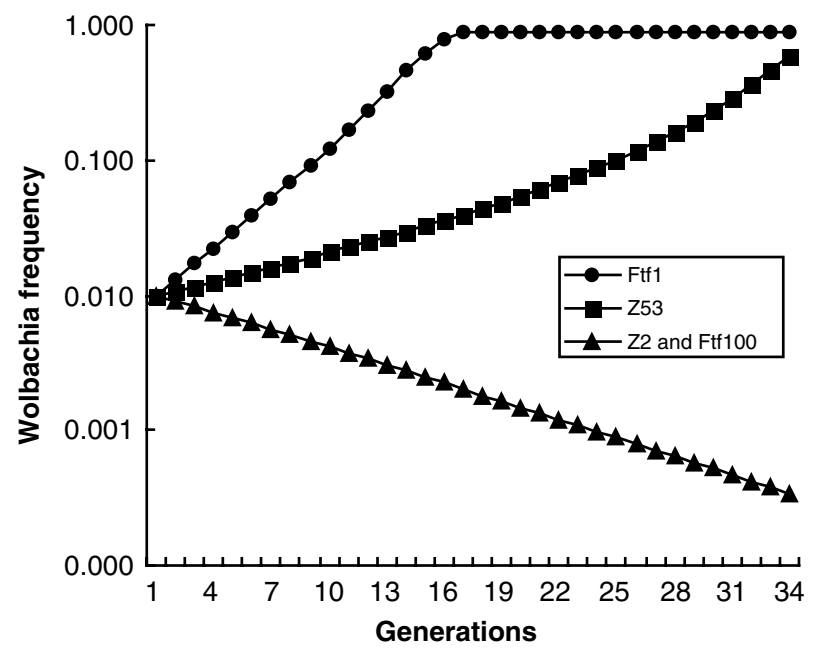

Figure 4 Cytoplasmic drive rates predicted for four D. melanogaster strains. Strain Ftf1, circle; strain Z53, square; and strains Ftf100 and $\mathrm{Z} 2$, triangle. The initial frequency was set to 0.01 . We used a 0.1 value for the fraction of uninfected eggs produced by an infected female (Olsen et al, 2001). The relative fecundity of uninfected females was determined by averaging crosses 1 and 3, then 2 and 4 , and comparing the averages (Table 3). Note the logarithmic scale of the ordinate axis.

addition, Snook et al (2000) found that infected $D$. simulans males produced fewer sperm cysts. In $D$. melanogaster, fitness is severely reduced by the virulent wDmpopcorn Wolbachia strain (Min and Benzer, 1997) that is different from the $w \mathrm{Dm}$ Wolbachia strain typically found in D. melanogaster and carried by our fly strains. Although wDmpopcorn and $\mathrm{wDm}$ share identical wsp sequences (McGraw et al, 2001), and both can negatively affect the fitness of $D$. melanogaster, they apparently affect fitness in different ways and are considered distinct Wolbachia strains (Min and Benzer, 1997). All our D. melanogaster strains carry the wDm Wolbachia strain, which is found in natural populations.

Treatment to remove Wolbachia bacteria produced no fitness response in fly strain Z2. Survival curves from infected and uninfected flies were not significantly different (Table 2), nor were there any differences in fecundity (Table 3), cytoplasmic incompatibility (Table 4), or egg to adult viability among the crosses (Table 5). Wolbachia's fitness effects have been documented in other D. melanogaster strains (Hoffmann et al, 1998; Olsen et al, 2001). In the latter study, small sample size and leastsquares analysis of mortality may have precluded the detection of significant survival differences (see Pletcher (1999) and references therein). We have recently demonstrated that the expression of Wolbachia-induced survival phenotypes can depend on inbreeding. For example, strain Z2, discussed in this report, shows no survival effect associated with Wolbachia; however, when Z2 flies are hybridized to Z53, infected F1 flies of both sexes survived significantly longer (Fry and Rand, 2002). Thus, the presence or absence of Wolbachia effects probably depends on other factors and more work is needed to determine which among those factors are most important.

Treatment to remove Wolbachia bacteria had a significant negative effect on the fitness of three $D$. melanogaster strains, Z53, Ftf1, and Ftf100. However, the positive fitness effects we observed were variable. For example, infected Z53 females showed better survival late in life, but seem to suffer heavier mortality early in life (Figure 1a). Lifetime fecundity measured from these Z53 females revealed no significant effect on Wolbachia infection (crosses 3 and 4 are not different). However, if one considers crosses 1 and 2 as well, then infected females lay more eggs than uninfected females regardless of the males' infection status. Although we did not directly measure male fertility, uninfected males might have better fertility than infected males because cross 2 was often more productive than cross 1 . We reported previously that Wolbachia could be associated with increased costs of reproduction in males from strains Z53 and Z2 (Fry and Rand, 2002). Wolbachia are known to interfere with sperm competition in Tribolium beetles (Wade and Chang, 1995), but similar effects have not been demonstrated in D. simulans or D. melanogaster (Hoffmann et al, 1998, 1990). Despite theoretical work showing that Wolbachia's effects should be concentrated in females (eg, Turelli, 1994), our data suggest there may be more Wolbachia effects in males than previously recognized (Fry and Rand, 2002) and more work is needed to determine the scope of Wolbachia's fitness effects in males.

Fly strains Ftf1 and Ftf100 also showed strong or variable positive fitness effects associated with Wolbachia infection. For example, strain Ftf100 showed no significant survival effect, but Wolbachia infection was associated with increased female fecundity during the earliest egg collection period (Table 3, time 1, crosses 2 and $4>$ crosses 1 and 3 ). However, this fecundity advantage was not evident on day 5 (Table 3, time 2). Unlike Z53 and Ftf100, only strain Ftf1 showed strong and persistent positive fitness effects associated with Wolbachia infection. Infected flies of both sexes survived significantly longer than uninfected flies (Figure 1b; Table 2). Infected females from Ftf1 also laid significantly more eggs during their lifetime, regardless of the male's infection status (ie, crosses 2 and $4>$ crosses 1 and 3; Table 3). In addition, Ftf1 was the only strain to show a significant difference in egg to adult viability (Table 5). Here cross 2 was greater than cross 3 . Our data provide compelling evidence for an association between Wolbachia bacteria and strong fitness effects in D. melanogaster. Consistent with our results, Olsen et al (2001) reported a dependence of Wolbachia-associated fecundity effects on the nuclear background of the host. Our data support a strong effect of host nuclear background and further suggest that Wolbachia's fitness effects can be strongly host-age dependent (Figure 3).

The fitness effects reported above are variable and appear to depend strongly on a number of factors including host nuclear background and gender, among other things. As an antibiotic treatment protocol is used to create uninfected fly stocks from infected fly stocks, there is a possibility that treatment itself could have produced the fitness effects we observed. Some previous studies of Wolbachia have included measures to guard against treatment effects. For example, Poinsot and Mercot (1997) used an uninfected treatment control in a study of Wolbachia fitness effects in D. simulans. They found no significant effect of tetracycline treatment on $D$. simulans fitness. In addition, Dedeine et al (2001) specifically tested the antibiotics tetracycline and 
rifampicin for fitness effects on the Wolbachia host Asobara tabida. Like Poinsot and Mercot (1997), Dedeine et al (2001) did not detect significant effects of antibiotic treatment on the fitness of their Wolbachia host. We also used an uninfected treatment control (RIredE) to test for effects of tetracycline treatment on D. melanogaster survival. There were no significant effects of tetracycline treatment on the survival of male or female RiRedE flies (data not shown). Two other sources of error could compromise our conclusions. First, we cannot discount the possibility that tetracycline treatment could have killed other bacteria or important symbionts in our fly strains and that these symbionts acting alone or interacting with Wolbachia could have produced the genotype-specific fitness effects we observed. However, much more work is needed to determine the identity of potential symbionts living with $D$. melanogaster and whether these symbionts influence host fitness or are affected by antibiotic treatment. Second, tetracycline treatment could have caused $\mathrm{T}$ and $\mathrm{W}$ flies to become genetically differentiated. To minimize this, we used inbred fly stocks in these experiments. All our fly strains were started with single, gravid, females and have been in laboratory culture for several hundred generations with brother-sister mating. Uninfected fly stocks were created directly from the infected stocks by antibiotic treatment. To see if there were transcriptional differences created by the antibiotic treatment, in our strongest responding fly stock, Ftf1, we used Affymetrix cDNA microarrays to examine transcriptional differences between infected and uninfected females from strain Ftf1. There was no evidence that named loci involved in antibiotic tolerance or resistance were transcriptionally altered by tetracycline treatment two generations after antibiotic treatment. These data support our contention that the fitness effects we found were not due to antibiotic treatment, but are instead most likely due to the presence or absence of Wolbachia bacteria.

The experiments reported above demonstrate that certain strains of Drosophila melanogaster die faster and lay fewer eggs when treated with antibiotics to remove Wolbachia. These beneficial effects are not observed in all fly strains: some showed no fitness effects associated with Wolbachia removal, while one strain lived longer when cleared of Wolbachia. None of the strains we studied exhibited CI, and this was confirmed at the earliest ages for nearly all of the fly strains. Since CI is weak or absent in D. melanogaster and previous studies have not detected clear fitness benefits of infection, the maintenance of Wolbachia in D. melanogaster remains unexplained (Hoffmann et al, 1994, 1998; Solignac et al, 1994; Olsen et al, 2001). Based on these observations and the experiments reported here, we suggest that Wolbachia might be maintained in some D. melanogaster populations by host-symbiont interactions that produce positive fecundity or longevity effects.

The positive fitness effects associated with Wolbachia infection in D. melanogaster could help promote the spread of infection throughout a host population. Figure 4 plots the cytoplasmic drive rates predicted for the four fly strains used in this study. These data are based on the model of Dobson et al (2002), except we substitute the relative fecundity from the incompatible cross (cross 1) instead of the relative hatch rate. We did this because there are no differences in egg hatch among our crosses, but there are differences in fecundity, and the two can be modeled similarly. Figure 4 shows that, for strains Ftf1 and Z53, a fecundity difference between T and $W$ flies is sufficient to promote an increase in Wolbachia infection without the need for CI induction. The Wolbachia infection in strains Ftf100 and Z2, however, has a neutral effect, and Wolbachia are lost from the host population due to the high rate $(10 \%)$ of incomplete maternal transmission used in the model. This figure is intended to illustrate our main finding that Wolbachia infection in D. melanogaster can produce a variety of fitness effects that can both promote and hinder the spread of infection. Although these models have worked very well in $D$. simulans, which has Wolbachia that express strong CI (Turelli and Hoffmann, 1995), the results presented here and elsewhere (Fry and Rand, 2002) indicate that models assuming a fitness equivalence for all matings (ie, crosses 1-4) may not be appropriate. Symbioses between Wolbachia and their hosts may be further complicated by evidence for considerable involvement of host nuclear genes in the expression of Wolbachia phenotypes (eg, Poinsot et al, 1998; Bordenstein and Werren, 2000; McGraw et al, 2001; Olsen et al, 2001; this report). Although the competing interests of Wolbachia and such host resistance genes have been modeled previously (eg, Randerson et al, 2000), more empirical work is needed to determine which host genes might be involved.

Our findings have implications for the evolution of the Drosophila-Wolbachia symbiosis. For vertically transmitted symbionts, natural selection will favor hosts that can suppress any parasitic phenotypes imposed by the symbiont. Similarly, selection will favor parasites that evolve traits ensuring their transmission into the next generation. Much of the literature on Wolbachia in Drosophila has focused on cytoplasmic incompatibility in D. simulans. In this species, Wolbachia achieves enhanced transmission by effectively suppressing the reproduction of uninfected females. That CI is weak or absent in $D$. melanogaster has been taken as indirect evidence for a longer history of association between Wolbachia and this host (Solignac et al, 1994). This argument rests on evolutionary arguments that hostparasite evolution under vertical transmission should lead to more benign parasitic effects, and eventually to mutualistic interactions (Anderson and May, 1982; Bull et al, 1991; Turelli, 1994). Our study of the Wolbachia-D. melanogaster symbiosis provides some of the first direct evidence for these predicted transitions between parasitic and mutualistic interactions. The Wolbachia-Drosophila symbiosis further provides a system where the phylogeny of the host species is well defined, but the hosts exhibit variation in the degree of parasitic and mutualistic phenotypes. As variation in these phenotypes is also present within species, this system offers excellent material for investigating evolutionary transitions between parasitic and mutualistic symbiont lifestyles.

\section{Acknowledgements}

We thank S Chein, J Greer, T Sackton, and L Sheldahl for help in the laboratory. M Tatar and S Pletcher provided 
useful discussion regarding demography cages and mortality analyses. This research was supported by NSF grant DEB-0104912 to AJF and grants DEB9527709, DEB-9981497, and INT-9981452 to DMR.

\section{References}

Anderson RM, May RM (1982). Coevolution of hosts and parasites. Parasitology 85: 411-426.

Anne-Veronique T-D, Raoult D, Drancourt M (2003). RNA polymerase Beta subunit-based phylogeny of Ehrlichia spp. Anaplasma spp., Neorickettsia spp. and Wolbachia pipientis. Int J System Evol Microbiol 53: 455-458.

Bandi C, McCall JW, Genchi C, Corona S, Venco L, Sacchi L (1999). Effects of tetracycline on the filarial worms Brugia pahangi and Dirofilaria immitis and their bacterial endosymbionts Wolbachia. Int J Parasit 29: 357-364.

Bordenstein SR, Werren JH (1998). Effects of A and B Wolbachia and host genotype on interspecies cytoplasmic incompatibility in Nasonia. Genetics 148: 1833-1844.

Bordenstein SR, Werren JH (2000). Do Wolbachia influence fecundity in Nasonia vitripennis. Heredity 84: 54-62.

Braig HR, Zhou W, Dobson S, O'Neill SL (1998). Cloning and characterization of a gene encoding the major surface protein of the bacterial endosymbiont Wolbachia pipientis. J Bacteriol 180: 2373-2378.

Bull JJ, Molineux IJ, Rice WR (1991). Selection of benevolence in a host-parasite system. Evolution 45: 875-882.

Caspari E, Watson GS (1959). On the evolutionary importance of cytoplasmic sterility in mosquitoes. Evolution 13: 568-570.

Chapman T, Miyatake T, Smith HK, Partridge L (1998). Interactions of mating, egg production and death rates in females of the Mediterranean fruit fly, Ceratitis capitata. Proc Roy Soc Lond B 265: 1879-1894.

Clancy DJ, Hoffmann AA (1998). Environmental effects on cytoplasmic incompatibility and bacterial load in Wolbachia-infected Drosophila simulans. Entomol Exper Applic 86: 13-24.

Cox DR (1972). Regression models and life tables. J Roy Stat Soc 34: 187-202.

Dedeine F, Vavre F, Fleury F, Loppin B, Hochberg ME, Bouletreau M (2001). Removing symbiotic Wolbachia bacteria specifically inhibits oogenesis in a parasitic wasp. Proc Natl Acad Sci USA 98: 6247-6252.

Dobson SL, Marsland EJ, Rattanadechakul W (2002). Mutualistic Wolbachia infection in Aedes albopictus: accelerating cytoplasmic drive. Genetics 160: 1087-1094.

Fine PEM (1978). On the dynamics of symbiote-dependent cytoplasmic incompatibility in Culicine mosquitoes. J Invertebr Pathol 30: 10-18.

Fowler K, Partridge L (1989). A cost of mating in female fruit flies. Nature 338: 760-761.

Fry AJ, Rand DM (2002). Wolbachia interactions that determine Drosophila melanogaster survival. Evolution 56: 1976-1981.

Hoffmann AA, Clancy DJ, Merton E (1994). Cytoplasmic incompatibility in Australian populations of Drosophila melanogaster. Genetics 136: 993-999.

Hoffmann AA, Hercus M, Dagher H (1998). Population dynamics of the Wolbachia infection causing cytoplasmic incompatibility in Drosophila melanogaster. Genetics 148: 221-231.

Hoffmann AA, Turelli M (1988). Unidirectional incompatibility in Drosophila simulans: inheritance, geographic variation and fitness effects. Genetics 119: 435-444.

Hoffmann AA, Turelli M, Harshman LG (1990). Factors affecting the distribution of cytoplasmic incompatibility in Drosophila simulans. Genetics 126: 933-948.
Hoffmann AA, Turelli M, Simmons GM (1986). Unidirectional incompatibility between populations of Drosophila simulans. Evolution 40: 692-701.

Jeyaprakash A, Hoy MA (2000). Long PCR improves Wolbachia DNA amplification: wsp sequences found in $76 \%$ of sixty-three arthropod species. Insect Mol Biol 9: 393-405.

Lipsitch M, Nowak MA, Ebert D, May RM (1995). The population dynamics of vertically and horizontally transmitted parasites. Proc R Soc Lond B 260: 321-327.

McGraw EA, Merritt DJ, Droller JN, O'Neill SL (2001). Wolbachia-mediated sperm modification is dependent on the host genotype in Drosophila. Proc $R$ Soc Lond $B$ 268: 2565-2570.

Min KT, Benzer S (1997). Wolbachia, normally a symbiont of Drosophila, can be virulent, causing degeneration and early death. Proc Natl Acad Sci USA 94: 10792-10796.

Mueller LD, Nusbaum TJ, Rose MR (1995). The Gompertz equation as a predictive tool in demography. Exp Geront 30: 553-569.

Olsen K, Reynolds T, Hoffmann AA (2001). A field cage test of the effects of the endosymbiont Wolbachia on Drosophila melanogaster. Heredity 86: 1-7.

O'Neill SL, Giordano R, Colbert AM, Karr TL, Robertson HM (1992). 16S rDNA phylogenetic analysis of the bacterial endosymbionts associated with cytoplasmic incompatibility in insects. Proc Natl Acad Sci USA 89: 2699-2702.

Pletcher SD (1999). Model fitting and hypothesis testing for age-specific mortality data. J Evol Biol 12: 430-439.

Pletcher SD, Khazaeli AA, Curtsinger JW (2000). Why do life spans differ? Partitioning mean longevity differences in terms of age-specific mortality parameters. J Gerontol 55: B381-B389.

Poinsot D, Bourtzis K, Markakis G, Savakis C, Mercot H (1998). Wolbachia transfer from Drosophila melanogaster into $D$. simulans: host effect and cytoplasmic incompatibility relationships. Genetics 150: 227-237.

Poinsot D, Mercot H (1997). Wolbachia infections in Drosophila simulans: does the female host bear a physiological cost? Evolution 51: 180-186.

Promislow DEL, Tatar M, Pletcher SD, Carey J (1997). Below threshold mortality and its impact on studies in evolutionary ecology. J Evol Biol 12: 314-328.

Randerson JP, Smith NCG, Hurst LD (2000). The evolutionary dynamics of male-killers and their hosts. Heredity 84: $152-160$.

Reynolds KT, Hoffmann AA (2002). Male age, host effects and the weak expression or non-expression of cytoplasmic incompatibility in Drosophila strains infected by maternally transmitted Wolbachia. Genet Res 80: 79-87.

Snook RR, Cleland SY, Wolfner MF, Karr TL (2000). Offsetting effects of Wolbachia infection and heat shock on sperm production in Drosophila simulans: analyses of fecundity, fertility and accessory gland proteins. Genetics 155: 167-178.

Solignac M, Vautrin D, Rousset F (1994). Widespread occurrence of the proteobacteria Wolbachia and partial cytoplasmic incompatibility in Drosophila melanogaster. C R Acad Sci Paris 317: 461-470.

Stouthamer R, Breeuwer AJ, Hurst GDD (1999). Wolbachia pipientis: microbial manipulator of arthropod reproduction. Annu Rev Microbiol 53: 71-102.

Turelli M (1994). Evolution of incompatibility-inducing microbes and their hosts. Evolution 48: 1500-1513.

Turelli M, Hoffmann AA (1995). Cytoplasmic incompatibility in Drosophila simulans: dynamics and parameter estimates from natural populations. Genetics 140: 1319-1338.

Vaupel JW, Yashin AI (1985). The deviant dynamics of death in heterogeneous populations. Soc Meth 15: 179-211.

Wade MJ, Chang NW (1995). Increased male fertility in Tribolium confusum beetles after infection with the intracellular parasite Wolbachia. Nature 373: 72-74. 
Weeks AR, Reynolds KT, Hoffmann AA (2002). Wolbachia dynamics and host effects: what has (and has not) been demonstrated? Trends Ecol Evol 17: 257-262.

Werren JH (1997). Biology of Wolbachia. Ann Rev Ent 42: 587-609.

Werren JH, Guo LW, Zhang W (1995a). Evolution and phylogeny of Wolbachia: reproductive parasites of arthropods. Proc R Soc Lond B 261: 55-71.
Werren JH, Windsor DM, Guo LR (1995b). Distribution of Wolbachia among neotropical arthropods. Proc $R$ Soc Lond B 262: 197-204.

Wu C-I, Hollocher H, Begun DJ, Aquadro CF, Xu Y, Wu M (1995). Sexual Isolation in Drosophila melanogaster: a possible case of incipient speciation. Proc Natl Acad Sci USA 92: 2519-2523. 